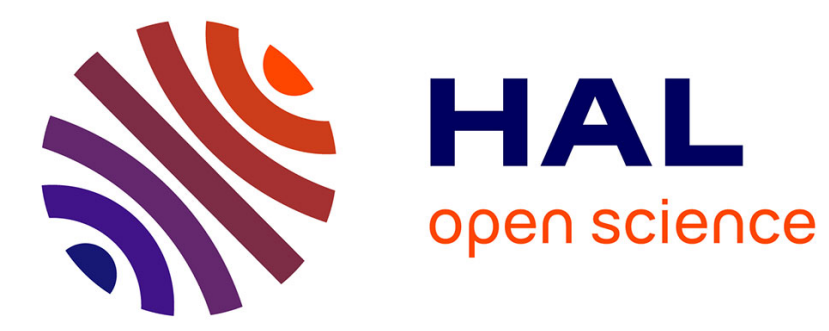

\title{
Thermo-fluorescent images of electric and magnetic near-fields of a High Impedance Surface
}

Hugo Ragazzo, Stéphane Faure, Daniel Prost, Julian Carrey, François Issac, Jean-François Bobo

\section{- To cite this version:}

Hugo Ragazzo, Stéphane Faure, Daniel Prost, Julian Carrey, François Issac, et al.. Thermo-fluorescent images of electric and magnetic near-fields of a High Impedance Surface. EMC Europe 2019, Sep 2019, BARCELONE, Spain. hal-02364970

\section{HAL Id: hal-02364970 https://hal.science/hal-02364970}

Submitted on 15 Nov 2019

HAL is a multi-disciplinary open access archive for the deposit and dissemination of scientific research documents, whether they are published or not. The documents may come from teaching and research institutions in France or abroad, or from public or private research centers.
L'archive ouverte pluridisciplinaire HAL, est destinée au dépôt et à la diffusion de documents scientifiques de niveau recherche, publiés ou non, émanant des établissements d'enseignement et de recherche français ou étrangers, des laboratoires publics ou privés. 


\section{Thermo-fluorescent images of electric and magnetic near-fields of a High Impedance Surface}

\author{
Hugo Ragazzo \\ ONERA/ DEMR \\ Université de Toulouse \\ F-31055Toulouse, France \\ hugo.ragazzo@1onera.fr \\ Stephane Faure \\ LPCNO \\ INSA \\ Toulouse, France \\ s-faure@insa-toulouse.fr
}

\author{
Daniel Prost \\ ONERA/ DEMR \\ Université de Toulouse \\ F-31055Toulouse, France \\ daniel.prost@onera.fr \\ Julian Carrey \\ LPCNO \\ INSA \\ Toulouse, France \\ julian.carrey@insa-toulouse.fr
}

\author{
François Issac \\ ONERA/ DEMR \\ Université de Toulouse \\ F-31055Toulouse, France \\ francois.issac@onera.fr \\ Jean-François Bobo \\ CNRS \\ CEMES \\ Toulouse, France \\ jean.francois.bobo@gmail.fr
}

\begin{abstract}
The characterization of the electromagnetic field emitted by various sources (antenna, radar...) is an important issue, either for civil or defense applications. The measurement of the electromagnetic field may be performed by a local probe, but the infrared thermography imaging is an alternative way. The latter method, called EMIR [1] (ElectroMagnetic InfraRed) has been used for years in particular at ONERA where it had been developed. We have recently successfully implemented this technique in the domain of visible light, named as EMVI [2]. As in the EMIR method, a thin film (sensitive to either electric field or magnetic field) is heated by the emitted field. But here the film is coated with a polymer doped with fluorescent molecules. As the fluorescent emission depends on the temperature of the film, we get a thermofluorescent sensor. The results presented here illustrate that new method: both magnetic and electric field imaging of a High Impedance Surface (HIS) has thus been obtained.
\end{abstract}

Keywords-Electromagnetism, thermography, magnetic losses, electric losses, fluorescence, High Impedance Surface.

\section{INTRODUCTION}

Microwave technologies play an important role in many fields from public applications (phone, communications) to more specific domains (radar, civil and military aeronautics, medicine...). In this context of increasing emissions of microwave electromagnetic fields, one of the major issues is to develop methods to diagnose and characterize it in real time.

We present in this paper the measurement of the electromagnetic near field of an High Impedance Surface (HIS) developed at ONERA [3]. We used a new method of measurement based on thermofluorescence. In the section II we enlighten this new concept called EMVI: ElectroMagnetic Visible Imaging. Then in section III we detail the specificity of this HIS. In the last section IV, images of the electric and magnetic near fields of the HIS are presented.

\section{EMVI TECHNIQUE}

The EMVI method uses a thin foil or film heated by the incident field [2, 3]. The foil is covered by fluorescent molecules which fluorescent emission depends on temperature. Hence, depending on the field amplitude, the film is more or less heated and the fluorescence intensity varies. This enables field imaging in the visible light domain. The interest of this technique is to be able to visualize and eventually to quantify the amplitude of a magnetic or electric field by a relatively non-intrusive way on the surface of a thin film that absorbs one part of the incident wave: a 2D cartography of the field can thus be obtained on a relatively large surface (several $\mathrm{dm}^{2}$ ), in a few seconds (much faster than a local probe that would be moved step by step to scan the whole surface). We could also get local measurements with the combination of an optical fiber and a thermofluorescent sensor for example for biological applications but this is out the scope of the present paper.

\section{A. Films with electric or magnetic losses}

Two types of microwave sensing films are used, either with electric or magnetic losses. These films have initially been developed for EMIR method. EMIR consists in imaging the electromagnetic field through the infrared emission of a thin film located in the field and heated by it. These thin films are polymer foils with small conductivity for electric field imaging or with ferromagnetic losses for magnetic film imaging. For the fluorescent EMVI method, the same films are coated with fluorescent molecules, as presented in the next section.

For electric losses we typically use $50-\mu \mathrm{m}$ thick Kapton film doped with carbone, with electrical conductivity $\sigma$ of about 10 to $100 \mathrm{~S} . \mathrm{m}^{-1}$. The conductivity is high enough to allow the formation of induced currents and therefore to heat the film as it is shown on Figure 1, but low enough to avoid electric absorption that would disturb and modify the incoming field.

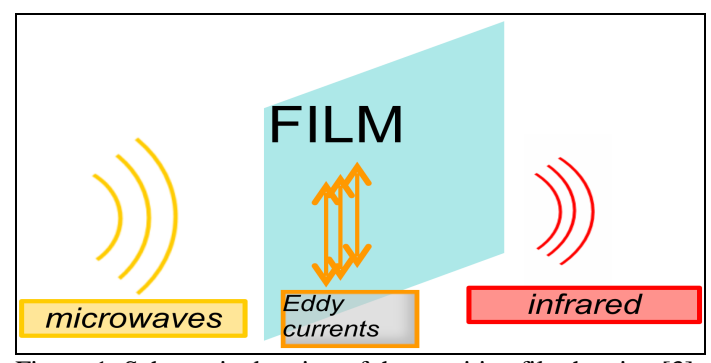

Figure 1: Schematic drawing of the sensitive film heating [3].

We want to absorb a small part of the electric field. The absorption rate of electric field, $A$, is given by: 


$$
A=\frac{4 Z_{S} Z_{0}}{\left(Z_{0}+2 Z_{S}\right)^{2}}
$$

With $Z_{0}=377 \Omega$ and $Z_{s}=1 /(\sigma \mathrm{e}) ; \sigma$ is the film electric conductivity and $\mathrm{e}_{1}$ its thickness.

The density of absorbed power $P_{\mathrm{abs}}\left(\mathrm{W} . \mathrm{m}^{-3}\right)$ is given by:

$$
P_{a b s}=\frac{2 Z_{S}}{\left(Z_{0}+2 Z_{S}\right)^{2}} E^{2}
$$

If $e$ (film thickness + fluorescence layer thickness) is small compared to the wavelength and penetration depth, the film heating at thermal equilibrium can be evaluated by:

$$
\Delta T_{\text {avg }}=\frac{P_{a b s} e}{2 h}
$$

Where $h$ is the heat transfer coefficient $\left(\mathrm{W} \cdot \mathrm{m}^{-2} \cdot \mathrm{K}^{-1}\right)$.

From (2) and (3) we obtain the electric field amplitude:

$$
|E|=k_{E} \sqrt{\Delta T_{\text {avg }}}
$$

with

$$
k_{E}=\left(\frac{2 \hbar}{g} * \frac{\left(z_{g}+2 z_{g}\right)^{2}}{2 a_{1} z_{S}}\right)^{1 / 2}
$$

Concerning the magnetic field component, the sensing film has to be both nonconductive and composed with high permeability elements (patterned ferromagnetic film as already published [4] or commercial FLEX-TOKIN absorber composed of ferromagnetic micro pellets in a polymer matrix [5]). In the microwave regime, ferromagnetic resonance (FMR) losses, or precession losses take place as we show on Figure $2 \mathrm{~b}$. These are related to the effective magnetic losses thickness defined by $t_{L}=\mu^{\prime \prime} . t$ (where $\mu^{\prime \prime}$ is the imaginary part of the permeability and $t$ its effective thickness). For lower frequencies heating is caused by hysteresis losses (Figure 2a).
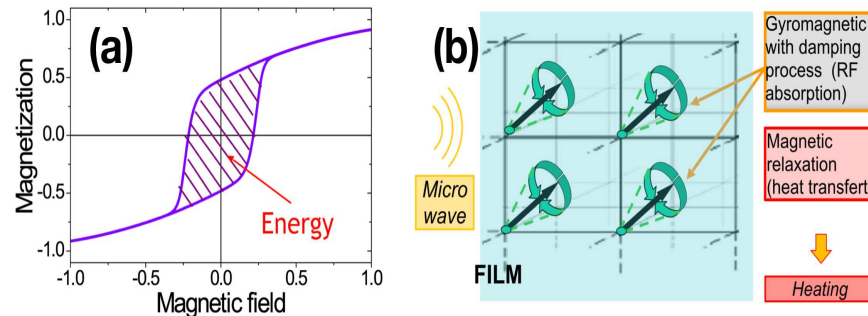

Figure 2: Sketches of hysteresis loop (a) Sketches of FMR (b)

The density of absorbed power $P_{\mathrm{abs}}\left(\mathrm{W} . \mathrm{m}^{-3}\right)$ is now:

$$
P_{a b g}=\pi f \mu^{\prime \prime} H^{2}
$$

From (3) and (6) we obtain the magnetic field amplitude:

$$
|H|=k_{H} \sqrt{\Delta T_{\text {avg }}}
$$

With

$$
k_{H}=\left(\frac{2 \pi e f \mu^{\prime \prime}}{c}\right)^{1 / 2}
$$

\section{B. Fluorescence loading}

A thin film of fluorescence solution is screen printed on a magnetic or electric losses film in clean room. The fluorescence film must be thinner than $100 \mu \mathrm{m}$ to neglect heat conduction. We have chosen rhodamine $\mathrm{B}(\mathrm{RhB})$ as the fluorophore. The absorption peak wavelength of Rhodamine B stirred in ethanol and epoxy is centered at $550 \mathrm{~nm}$ and the fluorescence emission peak is centered at $590 \mathrm{~nm}$. Our films are exposed to a $535 \mathrm{~nm}$ green light emitted by a LED array with a $450-550 \mathrm{~nm}$ band pass filter. To recover the fluorescence signal we have to use a high pass filter with cutoff wavelength at $580 \mathrm{~nm}$, we avoid any overlap between the green light excitation and the fluorescence signal. Absorption quantum yield of $\mathrm{RhB}$ in ethanol ranges from 0.69 to 0.97 [6]. We adapt the RhB molar concentration to maximize the fluorescence signal without $\pi$-stacking. Finally, the obtained fluorophore layer thickness is roughly $100 \mu \mathrm{m}$ on this sample (Figure 3).

Figure 3: TOKIN E25 + fluorescence RhB layer.

\section{Thermofluorescence setup}

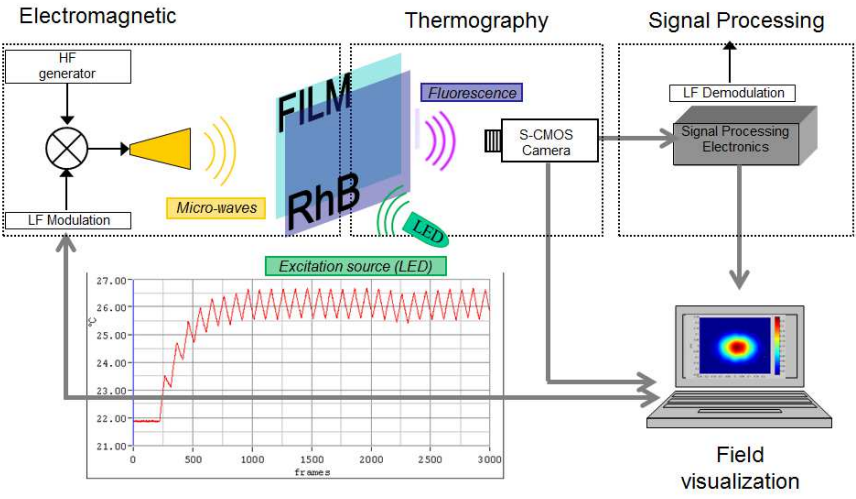

Figure 4: Thermofluorescense setup for measurement of the electromagnetic field

Figure 4 represents the schematics of the EMVI setup [7]. The microwave emitter is modulated (typically at $0.2 \mathrm{~Hz}$ ). The film is exposed to microwave modulated signal and LED excitation at $535 \mathrm{~nm}$ (green LED). Film heating is registered by the S-CMOS camera (Dhyana400D 4 megapixel). The advantage of the low frequency modulation is to cancel out all continuous effects (natural convection, thermal drift). Subsequent Fourier image demodulation allows increasing the signal-to-noise ratio.

\section{High IMPEDANCE SURFACE (HIS)}

A HIS is a special passive electromagnetic structure (based on metamaterial technology) designed for reducing mutual coupling between patch antennas (for applications where size constraints are crucial: space systems for example).

Where $C$ is the film heat capacity. 
(a)

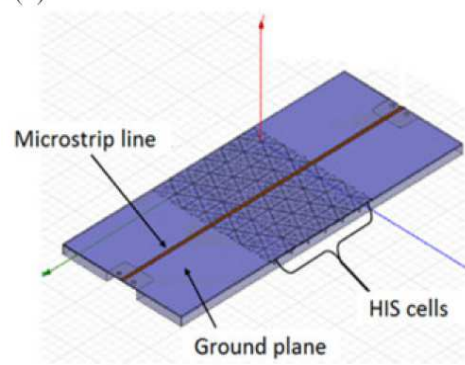

(b)

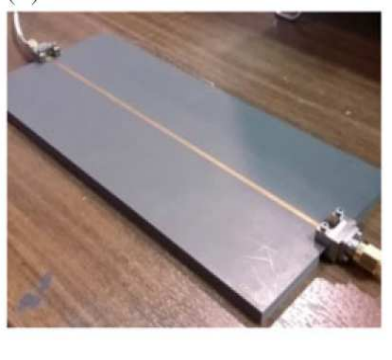

Figure 5: Simulation setup of the HIS embedded in the transmission line, (b) picture of the realized structure [8]

Figure 5(a) shows how the HIS cells are located between the ground plane and the transmission line [8]. It's made of periodic elements consisting of two layers dielectric substrate. The first dielectric layer includes a ground plane on the bottom face. The second layer is made of the same material than the first. This layer is printed on its two faces with copper. On the bottom we have hexagon shape and on the top, equilateral triangle shape with metallic via to connect the ground plane. This HIS is therefore composed of periodic capacitive and inductive elements. We can simply model HIS by a LC parallel circuit:

$$
Z(w)=\frac{j L w}{1-L C w^{2}}
$$

The first resonance frequency is given by:

$$
f_{0}=\frac{1}{2 \pi \sqrt{L C}}
$$

And the bandwidth is:

$$
B W \cong \sqrt{L C}
$$

Figure 6 shows S12 parameters, with and without the electric losses film [8]. We conclude from this picture that we have resonance peaks at $1.2 \mathrm{GHz}$ and a second one at about $1.9 \mathrm{GHz}$ (EMVI measurements will therefore be achieved at $2 \mathrm{GHz}$ ). It can also be noticed that the film is relatively non-intrusive in so far as the resonance peaks are almost not affected.

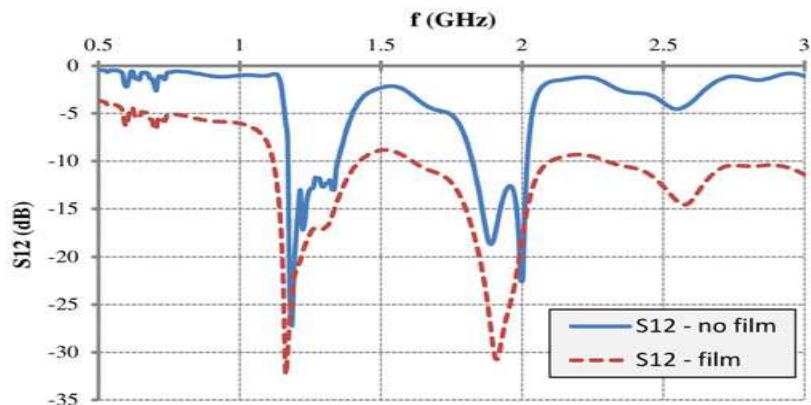

Figure 6: S12 measurement of the HIS, with and without the thermoemissive film [7]

\section{IMAGING NEAR FIELD OF HIS}

\section{A. Experimentation}

We use a green LED-array source made with 180 LEDs (at $535 \mathrm{~nm}$ ). The light source laid about $20 \mathrm{~cm}$ from the sample, and was tilted to avoid specular reflection (Figure 7). It provides an optical power density of $2.5 \mathrm{~mW} / \mathrm{cm}^{2}$ on the film surface. The camera is placed at zero-degree incidence with focal distance $20 \mathrm{~cm}$. The acquisition time is $1 \mathrm{~ms} ; 10$ images per second are collected for image processing (demodulation). The film is just in front of the HIS at about $1 \mathrm{~mm}(\lambda / 150)$.

The microwave power (modulated at $0.2 \mathrm{~Hz}$ ) is of the order of 2 to $3 \mathrm{~W}$. The HIS structure is connected to a $50 \Omega$ load. Measurements have been achieved for the $2 \mathrm{GHz}$ resonance frequency, and also at $2.25 \mathrm{GHz}$ to compare with the nonresonant behavior. Images are obtained in both configurations and given in the next section.

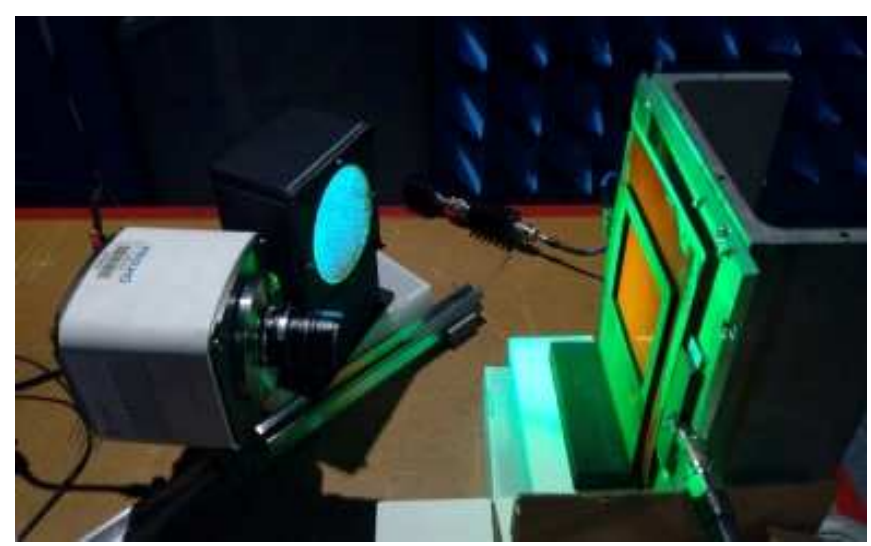

Figure 7: EMVI Setup in $\mathrm{H}$ field imaging mode.

\section{B. Measurement}

First, we observe the time dependence of one pixel intensity during microwave modulation on Figure 8. The signal-tonoise ratio of this signal has significant effect on the quality of the images. We applied the demodulation on all pixels to obtain images of the field.

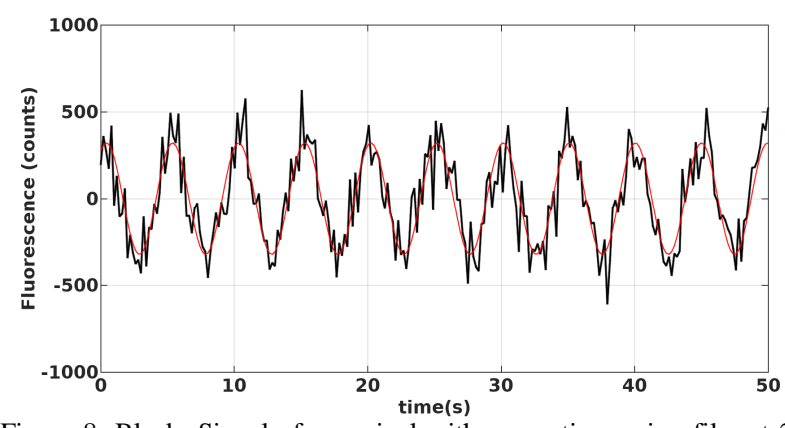

Figure 8: Black: Signal of one pixel with magnetic sensing film at $2 \mathrm{GHz}$ [X=450 Y=170], Red: sine fit of the signal.

On Figure 8 the signal of one pixel is centered and rectified. As we can see we have a good correlation with sine fit. The fluorescence variation is extracted from the signal by demodulation at the magnetic field modulation frequency deduced from the fit of one pixel. The amplitude of magnetic and electric field is proportional to the square root of the heating according to equations (4) and (7). Figure 9, 10,11 and 12 are images of the magnetic and electric fields, 
for two frequencies. We don't use $k_{h}$ and $k_{\mathrm{e}}$ coefficient so all images are in arbitrary unit (a.u).

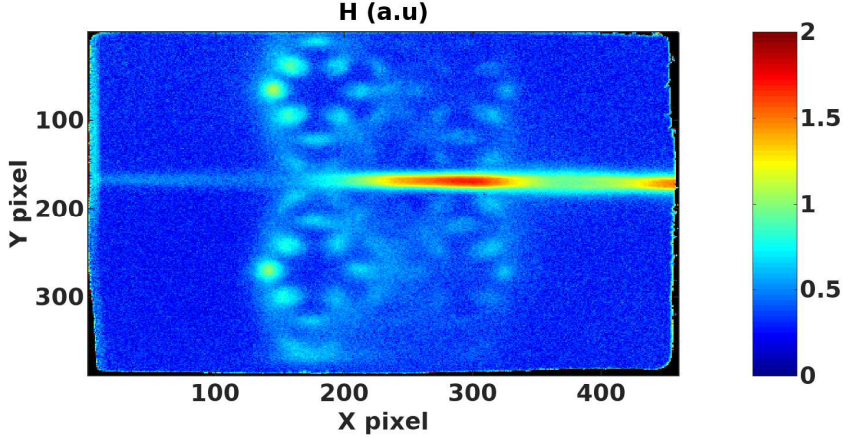

Figure 9: Magnetic near field image of HIS at $2 \mathrm{GHz}$

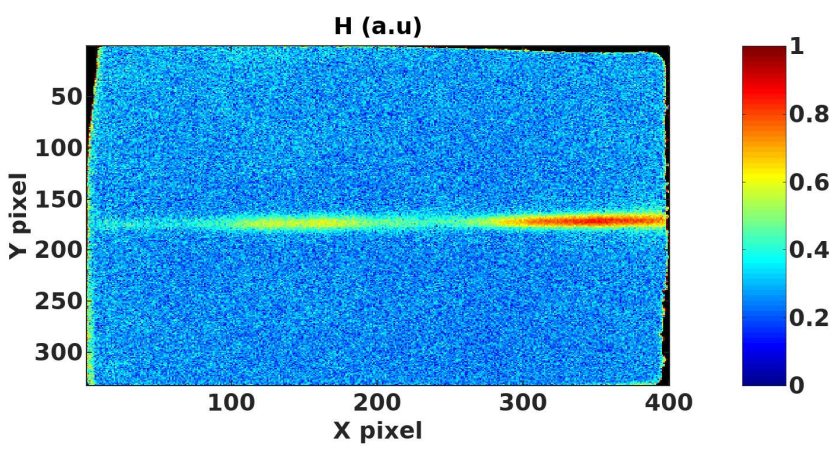

Figure 10: Magnetic near field image of HIS at $2.25 \mathrm{GHz}$

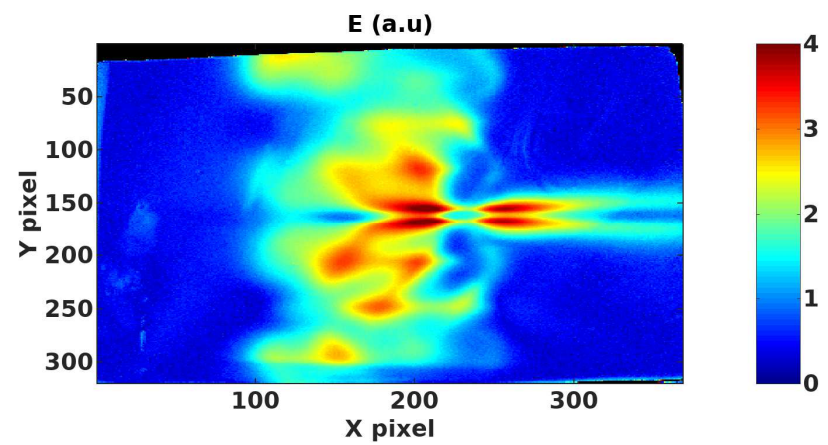

Figure 11: Electric near field image of HIS at $2 \mathrm{GHz}$

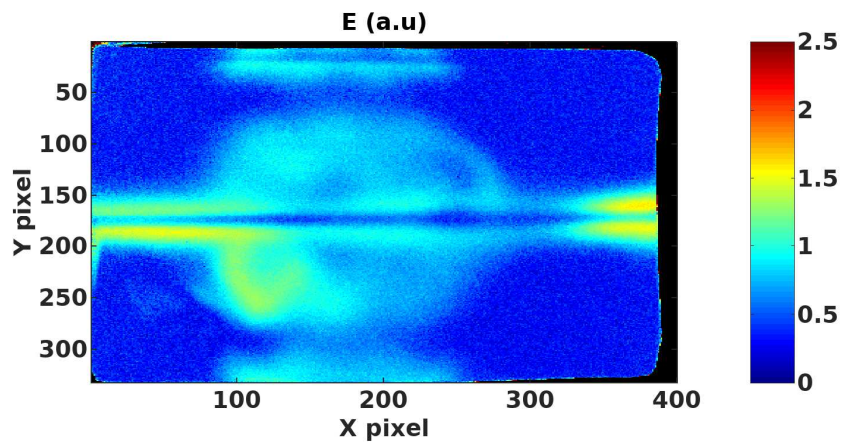

Figure 12: Imaging electric near field of HIS at $2.25 \mathrm{GHz}$

These images show very different behaviors of the HIS depending on the frequency:

At $2 \mathrm{GHz}$ the HIS cells resonate and we can observe subwavelength details of the magnetic and electric near field (Figures 9 and 11). These details would have been very difficult to visualize using a standard probe, which illustrates the interest of the EMVI method.
At $2.25 \mathrm{GHz}$ the HIS is out of the band gap as we show Figures 10 and 12 therefore show a passing behavior without any resonance. On Figure 12 we see we are not completely out of the resonance.

\section{CONCLUSION}

The EMVI method gives encouraging results on the HIS structure. It could be difficult to obtain large-scale images of magnetic or electric field with local probes. EMVI allows obtaining those images in a few seconds. Using simulations would cost more time and effort. Compared to the similar EMIR method, the main advantages of EMVI are the low cost of the setup and the spatial resolution of S-CMOS camera, which is better than any IR camera. Improvement of the method is under progress. EMVI is proposed as a new thermo-optic imaging sensor for electromagnetic compatibility.

\section{ACKNOWLEDGMENT}

This work was partly supported by NEXT Laboratory of Excellence (Université de Toulouse, CNRS) and CNRS Interdisciplinary Mission DEFINSTRUM.

\section{REFERENCES}

[1] P.Levesque, L.Leylekian, "Capteur vectoriel de champs électromgnétique par thermographie infrarouge”, French Patent $\mathrm{n}^{\circ} 9816079,1998$

[2] S. Faure, J.F. Bobo, J. Carrey, F. Issac and D. Prost, " Composant sensible pour dispositif de mesure de champ électromagnétique par thermofluorescence, procédés de mesure et de fabrication correspondants ", French Patent, ${ }^{\circ}$ 1758907, 2017

[3] N.Capet, "Amélioration du découplage inter élément par surface haute impédance pour antennes réseaux GNSS compactes », Ph.D. Report, Universit'e de Toulouse, 2010 [in french]

[4] J.Vernieres, J.F. Bobo, D. Prost, F. Issac, F. Boust, "Ferromagnetic microstructured thin films with high complex permeability for microwave applications", J. of Appl. Phys. 109, 07A323, 2011.

[5] https://www.tokin.com/english/product/pdf_dl/flex.pdf

[6] R.F.Kubin and A.N.Fletcher, "Fluorescence quantum yields of some Rhodamine dyes”, Journal of Luminescence 27 (1982) 455-462

[7] H.Ragazzo, D.Prost, JF. Bobo, S.Faure, J.Carrey, F.Issac, "Detection and imaging of magnetic field in the microwave regime with a combination of magnetic losses material and thermofluorescent molecules", IEEE Transactions on Magnetics (Volume: 55, Issue: 2, Feb.2019)

[8] D.Prost, F.Issac, C.Martel, N.Capet, J.Sokoloff, O.Olivier, "Electric field imaging of a high impedance surface for GNSS array decoupling application”, Eur. Phys. J. Appl. Phys. (2015) 